\title{
True Cause for Multi-Layer Inductive Coils' Sensitivity to Ambient Relative Humidity
}

\author{
Ling-Xiang Liu ${ }^{1}$, Zhong-Tai Qian ${ }^{2}$ \\ ${ }^{1}$ Retired from National Metrology Centre of Singapore since Nov. 2008, Corneliusstr. 12, 10787, Berlin, Germany, \\ liulingxiang08@gmail.com \\ ${ }^{2}$ Retired from National Institute of Metrology, 100013, Beijing, China
}

\begin{abstract}
For analyzing the properties of humidity-effect on the measurement of the inductance of multi-layer coils, a formula that establishes an approximate relationship between the coil's inherent capacitance and interlayer parasitic capacitance has been derived. Such a derivation using the inductor's equivalent circuit to include humidity effects clarifies the characterization of inductance, after more than half a century of neglect in the literature.
\end{abstract}

Keywords: Equivalent circuit, revelation, true cause, neglect for 65 years, humidity-effect, multi-layer inductive coils.

\section{INTRODUCTION}

As long as the subject "humidity influence on inductance measurement" is mentioned, almost every expert in this field unanimously recommends [1] as a classic, only on account of "coil dimensional change". In fact, the authors' purpose of introducing "coil dimensional change" is: "In order to obtain more information on the cause of the inductance changes particularly in view of the different behavior of the single layer $100 \mu \mathrm{H}$ inductor and that of the multi-layer inductors" [1]. However, the "coil dimensional change" does not give the true cause for the above-mentioned "different behavior".

Actually, the authors of [1] already clearly revealed the humidity change influence on the inductance measurement of multi-layer inductive coils in the magnitude aspect. It is shown in the Conclusions:

"It is of interest to note that except on the $100 \mu \mathrm{H}$ inductor the changes of inductance with relative humidity are about the same magnitude as the changes of capacitance in an air capacitor due to the change in permittivity of air: this is an increase of 0.2 in 10000 for a $10 \%$ rise in relative humidity".

Reference [1] was published in 1956 and it has been 65 years since then. Unfortunately, the authors' profound insight into the humidity effect has been often ignored. Nevertheless, after consideration, there are still some questions worth studying carefully: where is the so-called "air capacitor", and can its capacitance be measured, or approximately estimated? So far, these questions still cannot be clearly answered, but the dimensional changes of the coils induced by humidity changes have been indeed assumed to be the main cause, even forming a wide-spread misunderstanding in the metrology community.

Frequently, the key to resolving a problem lies in the problem itself. Why "multi-layer coils are sensitive to humidity amid inductance measurements" should be due to the "multi-layer" itself : with considerable parasitic capacitances among layers. Using the inductor's equivalent circuit to include humidity effects could clarify the characterization of inductance and simplify the analysis process greatly.

In 1883, French telegraph engineer L. C. Thévenin extended Ohm's law to the analysis of complex electrical circuits, until terahertz antennas, the hot new research topic nowadays, equivalent circuit has shown the simplest form of a more complex circuit in order to aid the property analysis. Accordingly, after doing some necessary concept preparations in Section 2 and Section 3, a formula that establishes an approximate relationship between the inherent capacitance of a multi-layer inductive coil and its interlayer parasitic capacitance is derived in Section 4. Relying on the true cause for the multi-layer coils' sensitivity to humidity we can quantify the static and dynamic characteristics via the exponential decay diffusion of water vapor followed by corresponding variations of electromagnetic features of air, as done in references [2]-[4]. Finally, the conclusions are drawn in Section 5. 


\section{EQUIVALENT CIRCUIT OF AN INDUCTOR WITH PARASITIC PARAMETER}

References [2]-[4] illustrate the effects of varying humidity behaviors in several electromagnetic measurement situations. These references quantify the exponential decay diffusion of water vapor under varying electromagnetic conditions. Applying these analyses to inductance coils with parameters, Fig.1. shows an ordinarily adopted equivalent circuit of an inductor with parasitic parameters.

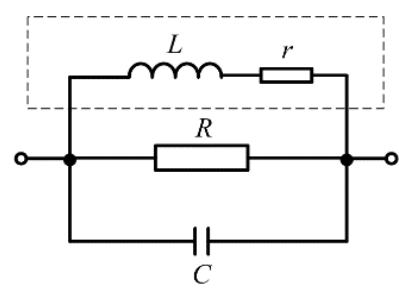

Fig.1. Equivalent circuit of an inductor with equivalent parasitic capacitance and equivalent leakage resistance.

In an ac inductance measurement at an angular frequency $\omega$, the obtained effective inductance value $L_{\text {eff }}$ of the measured inductor is given by

$$
L_{e f f}=L \frac{\left(1-\omega^{2} C L-C \frac{r^{2}}{L}\right)}{\left(1+\frac{r}{R}-\omega^{2} C L\right)^{2}+\omega^{2}\left(\frac{L}{R}+r C\right)^{2}}
$$

where $L$ is the inherent inductance, $C$ the equivalent parasitic capacitance, $R$ the equivalent leakage shunt resistance, and $r$ the internal resistance of the measured inductor, as shown in Fig.1. Ignoring the high order and cross-function small quantities, $L_{\text {eff }}$ can be simplified as

$$
L_{e f f} \approx L\left(1+\omega^{2} C L-\frac{2 r}{R}-\frac{C r^{2}}{L}\right)
$$

For a high inherent inductance value $L$, we have

$$
L_{e f f} \approx L\left[1+\omega^{2} C L-\frac{2 r}{R}\right]
$$

For a low inherent inductance, (2) can be simplified as

$$
L_{e f f} \approx L\left[1-\frac{C r^{2}}{L}-\frac{2 r}{R}\right]
$$

3. PARASITIC ADMITTANCE UNIFORMLY DISTRIBUTED BETWEEN TWO LAYERS OF WINDINGS, AND ITS EQUIVALENT ADMITTANCE

Regarding the external loops' characteristics of an $n$ terminal network, when the network is passive and reciprocal, the actual parasitic admittances in this network can be equivalently substituted by $n(n-1) / 2$ equivalent parasitic admittances, each of them being correspondingly connected between two outlet terminals. The most commonly used analysis objects are for $n=2,3$, or 4 .
Now let us study two layers of windings, which are evenly wound on the same annular skeleton, and the distribution of each layer of windings is around the circumference of this ring, the beginning and the end are exactly at the same position around the circumference. There is an angle $\alpha_{0}$ between the start-(end-) points of the two layers of windings. The total parasitic admittance uniformly distributed between the two layers of windings can be obtained to be $Y_{0}$ after actual measurement or calculation. We outline this winding principle in Fig.2.a), and its equivalently converted parasitic admittance in Fig.2.b).

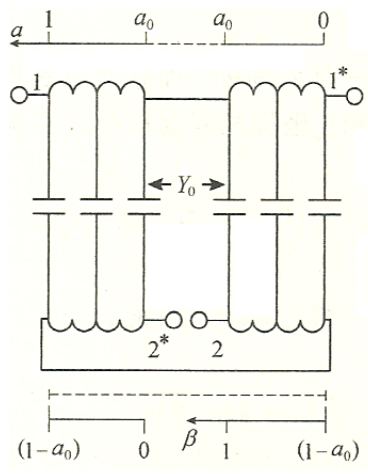

a) Parasitic admittance distribution

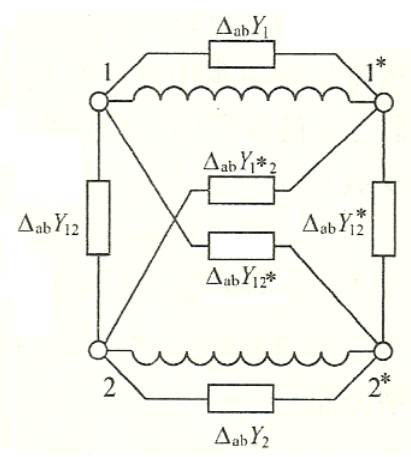

b) Equivalent admittance between two layers of windings
Fig.2. Parasitic admittance uniformly distributed between two layers of windings, and its equivalent admittance.

As shown in [5], [6], the conversion results are

$$
\begin{gathered}
\Delta_{a b} Y_{1}=\Delta_{a b} Y_{2}=-\frac{Y_{0}}{6} \\
\Delta_{a b} Y_{12}=\Delta_{a b} Y_{12} *=Y_{0}\left[\frac{1}{3}-\frac{\alpha_{0}\left(1-\alpha_{0}\right)}{2}\right] \\
\Delta_{a b} Y_{1 * 2}=\Delta_{a b} Y_{12^{*}}=Y_{0}\left[\frac{1}{6}+\frac{\alpha_{0}\left(1-\alpha_{0}\right)}{2}\right]
\end{gathered}
$$

\section{ESTABLISHING AN APPROXIMATE RELATIONSHIP BETWEEN INHERENT CAPACITANCE AND INTERLAYER PARASITIC CAPACITANCE OF A MULTI-LAYER COIL}

When a coil is made up of two layers of windings in series, its inherent capacitive admittance $Y$ can be obtained from the total interlayer parasitic admittance $Y_{0}$, as illustrated in Fig.3.

By means of the equivalent parasitic admittance conversion described in the previous section, we can obtain the inherent capacitive admittance $Y$ from the total parasitic admittance $Y_{0}$. Considering in this case two layers of windings in series, i.e. $\alpha_{0}=0$, as shown in Fig.3., $Y$ will be $Y_{0} / 4$.

For multi-layer coils (layers $\geq 2$ ), Fig.4. shows the schematic cross-sectional view and the parasitic interlayer capacitive admittances for the calculation.

As an example, Table 1. has listed the calculation results of the equivalent parasitic admittance converted to the two outlets for the number of winding layers up to 8 . For more layers, it can be calculated using ordinary admittance seriesparallel formulas as well. 


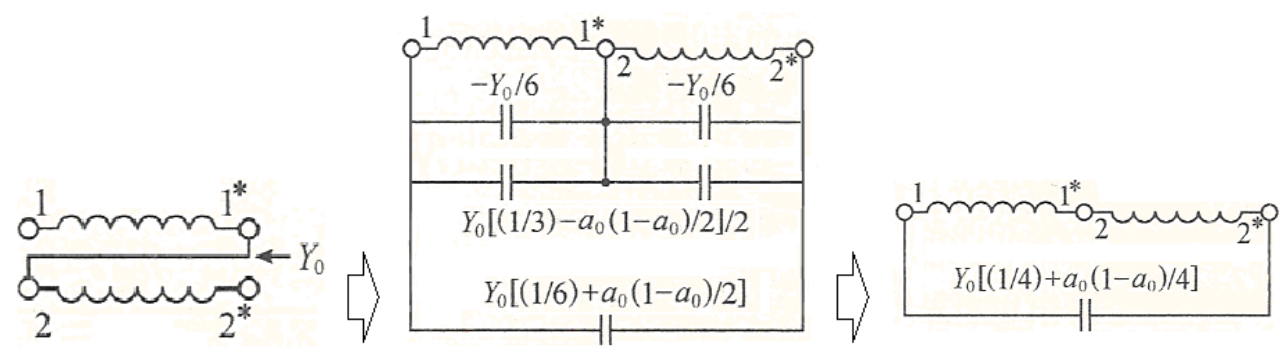

Fig.3. Derivation of inherent capacitive admittance $Y$ from total interlayer parasitic admittance $Y_{0}$ for a coil with two layers of windings in series via equivalent parasitic admittance conversion.

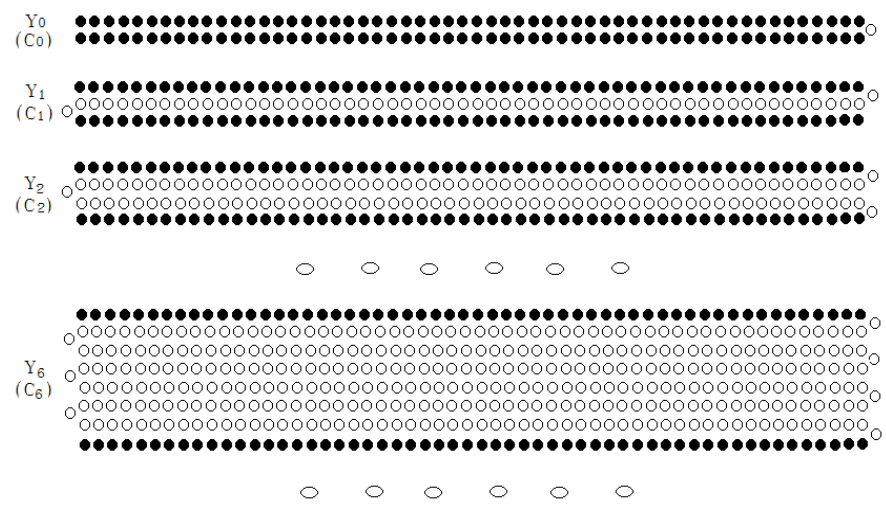

Fig.4. Schematic cross-sectional view of multi-layer coil and parasitic interlayer capacitive admittances (indicated by black solid circles) for calculation.

Table 1. Calculation results of the equivalent parasitic admittance converted to the two outlets of multi-layer coil (layers up to 8).

\begin{tabular}{|l|l|}
\hline Layers & Equivalent parasitic admittance converted to the two outlets \\
\hline 2 & $Y_{0} / 4$ \\
\hline 3 & $Y_{0} / 5+Y_{1} / 4$ \\
\hline 4 & $Y_{0} / 6+Y_{1} / 4+Y_{2} / 4$ \\
\hline 5 & $Y_{0} / 7+9 Y_{1} / 40+2 Y_{2} / 7+Y_{3} / 4$ \\
\hline 6 & $Y_{0} / 8+9 Y_{1} / 44+3 Y_{2} / 11+5 Y_{3} / 16+Y_{4} / 4$ \\
\hline 7 & $Y_{0} / 9+9 Y_{1} / 48+12 Y_{2} / 47+15 Y_{3} / 47+Y_{4} / 3+Y_{5} / 4$ \\
\hline 8 & $Y_{0} / 10+9 Y_{1} / 52+6 Y_{2} / 25+5 Y_{3} / 16+18 Y_{4} / 52+7 Y_{5} / 20+Y_{6} / 4$ \\
\hline
\end{tabular}

It can be clearly seen from Table 1 . that as the number of layers increases, the coefficients in front of $Y_{0}$ and $Y_{l}$ are both less than $1 / 4$, and the attenuations are relatively smaller. However, for the coefficients in front of $Y_{3}, Y_{4}$, and $Y_{5}$, all become larger than 1/4 and with relatively larger increments. The coefficient in front of $Y_{2}$ seems to be transitional, that is, it initially increases more than $1 / 4$, then diminishes, and afterwards is even less than $1 / 4$. If the number of layers is $>$ 8 such as up to 15,20 , or 30 , etc., the so-called "transitional" coefficient may appear in front of $Y_{3}, Y_{4}$, or $Y_{5}$, etc. In Table 1 , this potential trend has already emerged. Therefore, no matter how many layers $(\geq 2)$, using $Y_{0} / 4$ as the value of the parasitic capacitive admittance equivalent to the two outlets of a multi-layer inductor can approximate the sum of parasitic capacitive admittance terms with variable coefficients for different layers, as shown in Table 1.

Meanwhile, no matter how many layers $(\geq 2)$, this also means

$$
C \approx \frac{C_{0}}{4}
$$

where $C$ is the equivalent parasitic capacitance referred to the two outlets of a multi-layer inductor as shown in Fig.1., and $C_{0}$ is an often-measurable interlayer capacitance between two adjacent layers as illustrated at the top of Fig.4. (by black solid circles).

Thus, no matter how many layers $(\geq 2)$, the value of $C$ should be considerable. This is because $C_{0}$ is like the capacitance of a typical air plate capacitor, $C_{\text {air-plate }}=\varepsilon S / d$, here the plate area $S$ is large, $d$ as the distance between the two plates is very small, and the humidity effect depends mainly on $\varepsilon$, the permittivity of air. Often the value of $C_{0}$ can be measured, calculated, and estimated, with an acceptable accuracy, at any frequency within low-frequency range, and easy to do so.

Obviously, based on (6) the appearance described in the Conclusions of [1] can be clearly explained: regarding the response to humidity change amid measuring the inductance of a multi-layer inductive coil, the considerably large $C_{0}$ value makes the $C$-related terms in (2), (3), or (4) decisive. The formula (6) derived in view of equivalent circuit not only lets 
the study done 65 years ago [1] regain its deep insight, what it should really have, but also shows the measured inductance dynamic behavior of the multi-layer inductive coils after a humidity change: an exponential decay with time and having a longer time constant.

The appearance described in the Conclusions of [1] has revealed the humidity influence on measuring the inductance of multi-layer inductive coils only in the magnitude aspect. As for the behavior of measured inductance with time after a humidity change, i.e. the dynamic property, [1] only attempted to search in the way of "coil dimensional change" under extreme conditions, which are described in "Fig.3. Dimensional changes in a $2000 \mu \mathrm{H}$ inductor after eight days at $22 \%$ r.h." of [1]: exposing to $60 \%$ r.h "during a period of a month after it was taken from the container at $22 \%$ r.h." [1]. These specially arranged conditions are far beyond the maintenance conditions of a standard multi-layer inductive coil at any standard laboratory nowadays. The chosen way of "coil dimensional change" even failed to explain the typical behavior in "Fig.1. Variation of inductance in a $10000 \mu \mathrm{H}$ inductor with atmospheric relative humidity" of [1] by both static and dynamic aspects. This is because "coil dimensional change" can only lead to changes in the inherent inductance $L$ instead of "the changes of inductance with relative humidity are about the same magnitude as the changes of capacitance in an air capacitor due to the change in permittivity of air" [1].

However, using (6) does this integrated: the influence of varying humidity behaviors through the diffusion of water vapor with time exponential decay followed mainly by the change in permittivity of air, like a macro-relaxation process via moisture penetration into layers as a key interaction space. This behavior fully complies with the time-variant mathematical model, which was originally developed for describing the dependence of Zener voltage output on humidity [2], [4]. Unlike a pure mathematical step-response, the exponential decay diffusion of water vapor is also dependent on previous history of humidity change [2]-[4]. [1] has also a similar statement: "... show changes of inductance that depend upon the previous variations in the relative humidity".

In addition, it can also be seen from Table 1.: no matter how many layers $(\geq 2)$, moisture infiltration into (or out from) the outermost very thin gap, between the two adjacent layers, determines the time constants $\tau_{+}$and $\tau_{\text {- with time exponential }}$ decay behavior, which are to account for the fact that time constant is different depending on whether an ambient humidity change is positive or negative. The duration of time constant is determined by the sealing condition of such key interaction space from ambient atmosphere, usually $\tau_{-}>\tau_{+}$ [2]-[4].

The exception "on the $100 \mu \mathrm{H}$ inductor" mentioned in [1] is only a single-layer coil. The humidity influence on measuring such an inductance occurs mainly between its two binding posts. Therefore, the dynamic process is just like shown in "Fig.2. Variation of inductance of a $100 \mu \mathrm{H}$ inductor after two weeks at $22 \%$ r. h." of [1]: “...took place much more rapidly than with the other inductors mentioned above"; "An exponential curve with a time constant of about ten hours...", much shorter than that with multi-layer inductors, for instance with a $2000 \mu \mathrm{H}$ inductor, "between seven to ten days" [1]. On the other hand, since there is no longer considerable inter-layer parasitic capacitance in a single-layer coil, the amplitude of humidity change effect will depend on the combination of designed structure parameters and air-relevant parasitic parameters of the single-layer coil. This is the true cause for the "different behavior of the single layer $100 \mu \mathrm{H}$ inductor and that of the multi-layer inductors", not because of "coil dimensional change".

\section{CONCLUSIONS}

Measurement community mistakenly attributes the sensitivity of inductance to humidity to the dimensional change of the coil. However, the probability to have a coil dimensional change to multi-layer inductive coils at any standard laboratory, even though having some ambient relative humidity fluctuations, is small.

The analysis of humidity effects on multi-layer coils is achieved through derivation of a formula that establishes a relationship between the coil's inherent capacitance and interlayer parasitic capacitance. Thus, the unique static and dynamic behavior of multi-layer coils' sensitivity to ambient humidity, which was accurately observed 65 years ago, has shown its governing physics for the first time in that the true cause for the humidity-induced inductance change is actually the capacitance change between adjacent layers of the coils.

\section{REFERENCES}

[1] Rayner, G.H., Ford, L.H. (1956). The effect of humidity on the stability of inductance standards. In Journal of Scientific Instruments, 33, 75-78.

[2] Liu, L.X., Sim, T.Y., Tan, V.K.S., Chua, H.A., Lam, K.H. (2000). Mathematical model to approximate the response of a Zener cell output under varying environmental conditions. In Metrologia, 37, 213-218.

[3] Liu, L.X., Chua, S.W., Ang, C.K. (2002). High voltage AC-DC difference measurements under humidity step changes. In IEE Proceedings - Science Measurement and Technology, 149 (6), 317-319.

[4] Liu, L.-X., Li, Z., He, Q., Lu, Z., Zhang, Z. (2021). Common model to approximate the time-variant behaviour of air humidity-change influence on some fundamental standards of precision electromagnetic measurement. In Measurement, 171, 108647.

[5] Qian, Z.-T. (1965, 1975, 1977). The shielding and protection of electrical measurement circuits and apparatus. In Electrical Measurement and Instrumentation, 1-3 (1965), 3-12 (1975), 2-12 (1977). (in Chinese)

[6] Liu, L.-X., Qian, Z.-Y., Qian, Z.-T. (2002). Basic theory of shielding protection for electrical measurement circuits. In Chinese Metrology Press, Beijing. (in Chinese)

Received April 11, 2021 Accepted July 22, 2021 